\title{
Relative Therapeutic Efficacy of Phonophoresis and Cryotherapy as Combined Therapy in the Management of Musculoskeletal Injuries
}

\author{
Onuwe, H.A.K., ${ }^{1}$ Amadi, K. ${ }^{2}$ \\ ${ }^{1}$ Physiotherapy Department, Jos University Teaching Hospital, Jos \\ ${ }^{2}$ Human Physiology Department, University of Jos, Jos \\ Correspondence \\ Henry A.K. Onuwe, Physiotherapy Department, Jos University Teaching Hospital, Jos • Email: \\ raphaophysio@yahoo.com
}

\section{SUMMARY}

This study was designed to investigate the efficacy of phonophoresis and cryotherapy as combined therapy (double-modality therapy - DMT) in the management of pain among subjects with musculoskeletal injuries (MSIs).

Subjects were assigned randomly to one of three groups: D MT group (n=17) received cryotherapy and $15 \%$ methyl salicylate phonophoresis, PHONO group $(\mathrm{n}=14)$ received $15 \%$ methyl salicylate phonophoresis, and CRYO group $(\mathrm{n}=19)$ received cryotherapy and 'sham' phonophoresis. Ultrasound at an intensity of $1.5 \mathrm{~W} / \mathrm{cm}^{2}$ and frequency of $1 \mathrm{MHz}$ was used to apply methyl salicylate while intermittent cryotherapy was the mode of application. The pre- and post-treatment pain perception scores (PPS) of the patients were assessed using the visual analogue scale (VAS), and the sessions of treatment in all groups were recorded. Treatment was administered on alternate days and subjects in all groups were discharged when they were pain free.

In total, 233 treatment sessions were recorded - 61(26.2\%) in DMT, 77(33.0\%) in PHONO and 95(40.8\%) in CRYO group respectively, which indicated no significant difference $(P>0.05)$. In the DMT, CRYO and PHONO groups, 16, 12 and 7 subjects respectively were pain free after 1 to 5 treatments. The difference in the severity of pain was significant $(\mathrm{P}<0.05)$ in each group post-treatment, which suggests that DMT, phonophoresis and cryotherapy were equally effective.

The study has demonstrated the therapeutic efficacy of DMT, but it was not superior to the single treatment protocol of phonophoresis or cryotherapy. It might however, take less treatment sessions to reduce pain among the subjects with the use of DMT compared with other two modalities.

KEYWORDS: cryotherapy, double-modality therapy, musculoskeletal injury, phonophoresis, ultrasound

\section{INTRODUCTION}

Musculoskeletal injuries (MSIs), which are often due to strenuous activity (Hootman et al, 2002), account for roughly $25 \%$ of patient complaints in primary health care settings (Childs et al, 2005), and are a leading cause of work absenteeism worldwide (Bee et al, 2005). Also, musculoskeletal problems constitute the largest proportion of injuries among athletes (Reilly and Hardiker, 1983). A report has indicated that more than $60 \%$ of men and women who work on the computer have complained of pain resulting from various MSIs (Idowu et al, 2005). Low back pain (LBP) is not only one of the most common MSIs in industrialized societies (Sanya and Ogwumike, 2005; Saidu et al, 2011), it is the most costly, and the primary cause of disability in persons under 45 years (DeRosa and Porterfield, 1992). In the United States, more than 25,000 
ankle sprains occur each day and the concomitant symptoms (pains and swelling) leave the individuals with some functional disability (Man et al, 2007).

Physical treatments (i.e phonophoresis, cryotherapy, etc.) have been used over the years to reduce pain, control swelling or inflammation and improve or restore function in the management of MSIs (Bolin, 2003; Kozanoglu et al, 2003). Phonophoresis which is the use of ultrasound (US) to enhance percutaneous absorption of topical drugs in the management of MSIs (Machet and Boucuad, 2002; Kuntz et al, 2006) and dermatological conditions (Kozanoglu et al, 2003) has been a widely applied clinical therapeutic procedure (Byl, 1995; Kozanoglu et al, 2003) since the first time it was used in 1954 by Fellinger and Schmid to treat polyarthritis of the hand (Byl, 1995). It is believed to accelerate functional recovery by decreasing pain and promoting healing (Cagnie et al, 2003). In a comparative study involving iodex phonophoresis $(\mathrm{n}=15)$, iodex iontophoresis $(\mathrm{n}=15)$ and a placebo $(\mathrm{n}=15)$ in the management of shoulder periarthritis, Bumin and Can (2004) observed that iodex phonophoresis and iodex iontophoresis were significantly effective in decreasing pain as compared to the placebo. The application of cold (otherwise known as cryotherapy) for the treatment of injury or disease, particularly for increasing pain threshold, decreasing inflammatory reaction and spasm following MSIs (Swenson et al, 1996; Yagiz, 2006) has been advocated by some researchers as the sole treatment to be used during all phases of soft tissue injury (Cote et al, 1988; Swenson et al, 1996). In a systematic review of some randomized controlled trials (RCTs) to assess the efficacy of ice in the management of pain and swelling resulting from soft tissue injury, it is reported that cryotherapy seems to be effective in decreasing pain and speeds up return to full activity (MacAuley, 2001; Hubbard and Denegar, 2004).

Phonophoresis or cryotherapy in isolation or in combination with other therapies has become a widely used regimen for reducing pain, inflammation and improving or restoring function in the management of MSIs (Kellett, 1986; Palmer and Toombs, 2004; Wilson and Best, 2005). Davis (1991) recommended the application of cryotherapy as a single treatment protocol for hours (i.e. 72 hours) when there is trauma (i.e. rotator cuff syndrome) before resorting to phonophoresis for the remaining period of treatment. The literature has indicated that cold application prior to phonophoresis produces an intense hyperaemia which may improve the absorption and distribution of the medication to effect pain relief and resolution of inflammation (Santiesteban, 1983).

The recognition of the importance of pain control in recovery from MSIs to enable injured persons return to participation (athletes) or to work (typical population) has prompted clinicians to continue to explore more aggressive pain management strategies (Brolinson and Sampson, 2003; Hubbard et al, 2004). Some clinicians (Santiesteban, 1983; Balogun, 1990) have suggested that phonophoresis and cryotherapy can be combined (double-modality therapy DMT) in the management of MSIs for better outcome. Sequel to this, pain clinics and sport centres have adopted, as a tradition, the treatment protocol of combining phonophoresis and cryotherapy (DMT) in the management of MSIs worldwide. Despite the general acceptance and the frequency at which this treatment approach (DMT) is being practiced, there is a dearth in the literature to support the practice or demonstrate its efficacy or superiority over the single treatment protocol (Balogun, 1990; Ball, 2002). The practice is probably or largely based on anecdotal evidence rather than on empirical data. Clinical opinion alone is not good enough anymore. Hence, it is incumbent upon the clinician or the therapist to collect information or data to support clinical decision making with something more than physiologic philosophy based on opinion. Randomized clinical trials are required, therefore, to support clinical decision making on the use of phonophoresis and cryotherapy as a combined treatment protocol for optimal outcome or response following MSI management.

\section{METHODOLOGY}

\section{Subjects}

Fifty (50) subjects, comprising 37 men and 13 women aged 18 to 77 years with a mean of 34 years, who had sustained musculoskeletal injuries, were all recruited as they presented for treatment at the Jos University Teaching Hospital, Primary Health Centres and Sports Council Clinic; all located within Jos metropolis. Only subjects who sustained not more than one musculoskeletal injury (i.e. subjects with multiple injuries were not eligible) with acute onset of symptoms or occurring as an acute exacerbation of chronic lesion were included in the study. Subjects who were on any form of analgesic (steroids and non-steroidal anti-inflammatory drugs - NSAIDs), muscle relaxants and any form of physiotherapy treatment were all excluded from 
the study. Subjects with open wounds over the injury sites, pregnancy, disease conditions (e.g. thrombophlebitis, cardiac disease, patient with pacemaker, tumour, etc.) and those allergic to topical methyl salicylate or cold which contraindicate the treatment protocols used in this study were all excluded. The use of NSAIDs or analgesics or any other form of treatment was disallowed throughout the study period. Before entry into the study, each subject voluntarily signed the informed consent form, after the protocol for the trial had been explained. All procedures involving the subjects met the criteria established by the University of Jos Teaching Hospital Health Research Ethics Committee.

\section{Procedure}

On completion of history taking, and careful clinical and radiographic examination, the fifty (50) subjects whose conditions are classified as follows: rotator cuff syndrome(14), ankle sprain (7), knee sprain (4), patellar bursitis (4), low back pain (10), muscle contusion (3), muscle strain (4), tennis elbow (2), hamstring tendinitis (1), and De Quervain tenosynovitis (1), were randomly assigned to one of three treatment groups.

i. Double-modality Therapy ("Live" phonophoresis and cryotherapy combined) - DMT (17)

ii. "Live" phonophoresis only - PHONO (14)

iii. Cryotherapy and "sham" phonophoresis - CRYO (19)

These treatment groups were written and placed in sequentially numbered opaque sealed envelopes which were used to assign the subjects to their respective groups. Neither the primary researcher nor any other person that was involved in treatment allocation was aware of the randomization schedules (Brolinson and Sampson, 2003). The ultrasound machine (EMS Therasonic MK IV) and the transducer $\left(5 \mathrm{~cm}^{2}, 1 \mathrm{MHz}\right.$ and $3 \mathrm{MHz}$ treatment head) were all tested and certified functional. The subjects were carefully educated on the use of the VAS and it was observed that subjects could identify their pain levels or scores on the scale without any difficulties. Subjects pain perception was subjectively assessed or measured and recorded using a $10 \mathrm{~cm}$ visual analogue scale (VAS) marked " no pain" at one end and "worst pain ever" at the other (Klaiman et al, 1998; Hoppenrath and Ciccone, 2006). A sensory test was also conducted to ascertain that the subjecs had no sensory loss (Oakley, 1978). Subjects were made to understand that at no time during insonation (phonophoresis) should they suffer discomfort. There might be a sensation of very mild warmth, but other than that only the pressure and the movement of the transducer should be felt. They were instructed to report any other sensation at once. All subjects assigned to cryotherapy were made to understand that sensations such as cold, burning, aching and numbness would be felt during the treatment procedure, but they would not be harmful. Finally, subjects were comfortably supported and positioned to maximize circulation to the area being treated (Byl, 1995) when they were ready for treatment.

The subjects in the DMT group (17) received cryotherapy and "live" phonophoresis as combined therapy (DMT). Intermittent cryotherapy (MacAuley, 2001; Bleakley et al, 2006) using an ice pack $(16 \mathrm{~cm} \mathrm{x} 12 \mathrm{~cm})$ was applied directly over the subjects' affected areas for 10 minutes. The ice pack was then removed after the initial 10minute application. The treated part was then allowed to rest at room temperature for 10 minutes. The ice pack was reapplied immediately following the rest period for another 10 minutes (total cryotherapy period $=20$ minutes). At the expiration of the second ice pack application, the treated part was cleansed with a towel and continuous ultrasound at an intensity of $1.5 \mathrm{~W} / \mathrm{cm}^{2}$ and frequency of $1 \mathrm{MHz}$ (Byl, 1995; Klaiman et al, 1998) was used to apply $1.5 \mathrm{~g}$ of $15 \%$ methyl salicylate cream thoroughly mixed with $1.5 \mathrm{~g}$ of aquasonic gel (Allen, 2005) as coupling medium for 8 minutes. The ultrasound head was moved over the part under treatment about one and a half $\left(1 \frac{1 / 2}{2}\right)$ the width of the transducer at approximately 2 to $4 \mathrm{~cm} / \mathrm{sec}$; using small, continuous and overlapping circular movements (Oakley, 1978; Cagnie et al, 2003; Kuntz et al, 2006) to avoid or prevent periosteal pain (Santiesteban, 1983). These treatment values or settings were selected to capture both the thermal and nonthermal effects of ultrasound in order to optimize transdermal methyl salicylate $15 \%$ delivery (Byl, 1995; Cagnie et al, 2003).

The subjects in the PHONO group (14) received "live" phonophoresis as a single treatment protocol. The phonophoresis treatment procedure was exactly the same as applied in the DMT group. All subjects in the CRYO group (19) received cryotherapy and "sham" phonophoresis with a placebo coupling medium. Intermittent cryotherapy was applied directly over the subjects' affected areas with the same procedure described in the DMT group. At the 
expiration of cryotherapy application, the treated part was cleansed with a towel and continuous ultrasound at zero (0) intensity and frequency settings (no US transmission) was applied with $1.5 \mathrm{~g}$ of placebo aquasonic gel (devoid of methyl salicylate $15 \%$ cream) as coupling medium (Ciccone et al, 1991; McElnay et al, 2004) for 8 minutes.

Treatments were administered on alternate days in each group until subjects were fit for discharge. At the end of weeks 1, 2, 3 and 4 after treatment in each group, subjects' post-treatment pain perception scores (PPS) were assessed and recorded. Assessment and recording of pre- and posttreatment PPS were blinded from the researchers. Treatments were terminated and subjects discharged in all groups when subjects felt pain was sufficiently relieved and no longer needed treatment.

Descriptive and inferential statistics were carried out using the Statistical Package for the Social Sciences (SPSS) for data analysis. Independent and paired mean difference tests (t-test) were used to compute subjects' repeated measures within all groups, while a one-way ANOVA (Klaiman et al, 1998) was used to compute measures across the groups with the level of significance for all tests set at 0.05 .

\section{RESULTS}

The total number of treatment sessions (233) recorded in all the groups is presented in table 1 . Sixty-one $(26.2 \%)$ were recorded in the DMT group, 77 (33.0\%) in the PHONO group, while the CRYO group recorded $95(40.8 \%)$ sessions. The results in table 1 indicate that there was no statistically significant $(\mathrm{P}>0.05)$ difference in the mean treatment sessions between the three groups. The number of subjects discharged on or before completion of the treatment term in all the three groups is presented in table 2.

While all subjects were fit for discharge without necessarily completing the 12 treatment sessions initially designed for the study in the DMT group, $5.2 \%$ (1) of the subjects in the CRYO and $7.1 \%$ of the PHONO group received the full treatment sessions. Sixteen ( $94 \%$ ) subjects in the DMT group were fit for discharge after receiving treatment for 1 to 5 sessions. Only $7(50 \%)$ and $12(60 \%)$ subjects in the PHONO and CRYO groups respectively were fit for discharge after receiving 1 to 5 treatment sessions. While no subjects were fit for discharge in the PHONO group after receiving 1 to 2 treatment sessions, the
DMT and CRYO groups recorded 6 discharges each. Furthermore, when $10(58 \%)$ subjects were fit for discharge in the DMT group following 3 to 5 treatment sessions, the PHONO and CRYO groups recorded $7(50 \%)$ and $6(31 \%)$ respectively.

Table 1. Treatment sessions received by the subjects

\begin{tabular}{lccccc}
\hline Group & \multicolumn{5}{c}{ Treatment Sessions } \\
\cline { 2 - 6 } & Min. & Max. & Total & Mean \pm SD & P - value \\
\hline $\operatorname{DMT}(\mathrm{n}=17)$ & 2 & 9 & 61 & $3.59 \pm 1.87$ & $\mathrm{~F}=2.18$ \\
$\operatorname{PHONO}(\mathrm{n}=14)$ & 3 & 12 & 77 & $5.50 \pm 2.47$ & $\mathrm{P}>0.05$ \\
CRYO $(\mathrm{n}=19)$ & 2 & 12 & 95 & $5.00 \pm 3.37$ & \\
\hline
\end{tabular}

DMT $=$ double-modality therapy (phonophoresis + cryotherapy); PHONO $=$ phonophoresis; CRYO = cryotherapy

Min. = Minimum; Max. = Maximum;

$\mathrm{P}>0.05=$ No significant difference

Table 2. Subject discharge pattern

\begin{tabular}{lccc}
\hline $\begin{array}{l}\text { Sessions of } \\
\text { Treatment on } \\
\text { Discharge }\end{array}$ & $\begin{array}{c}\text { DMT } \\
(\mathrm{n}=17)\end{array}$ & $\begin{array}{c}\text { PHONO } \\
(\mathrm{n}=14)\end{array}$ & $\begin{array}{c}\text { CRYO } \\
(\mathrm{n}=19)\end{array}$ \\
\cline { 2 - 4 } & $6(35.3 \%)$ & 0 & $6(31.6 \%)$ \\
$1-2$ & $10(58.8 \%)$ & $7(50 \%)$ & $6(31.6 \%)$ \\
$6-5$ & $1(5.9 \%)$ & $6(42.9 \%)$ & $6(31.6 \%)$ \\
12 & 0 & $1(7.1 \%)$ & $1(5.2 \%)$ \\
\hline
\end{tabular}

$\mathrm{DMT}=$ double-modality therapy (phonophoresis + cryotherapy)

PHONO $=$ phonophoresis

CRYO = cryotherapy

Comparison of pre- and post-treatment PPS in each group (within group comparison) was made to determine if there was any significant difference in pain severity. The results are presented in table 3 . The difference in the severity of pain perception before and after treatment among the subjects in each group was statistically significant $(\mathrm{P}<0.05)$.

All pre-treatment PPS in all the groups were compared to ascertain any difference. The same was done for all the post-treatment PPS. The results are presented in table 4. There was no statistically significant difference $(\mathrm{P}>0.05)$ in pain perception before and after treatment among the subjects in all the groups. No subject complained of any discomfort or adverse effect, such as periosteal pain, skin 
allergy, frostbite or nerve palsy; instead the subjects reported that treatments were effective, tolerable and pleasant. All subjects felt satisfied with their level of pain relief and requested for discharge accordingly.

Table 3. Comparison of pain perception scores in each group

\begin{tabular}{|c|c|c|c|}
\hline \multirow[t]{2}{*}{ Group } & \multirow{2}{*}{$\begin{array}{l}\text { Pain } \\
\text { Assessment }\end{array}$} & \multicolumn{2}{|c|}{ Pain Perception Scores } \\
\hline & & Mean \pm SD & P-value \\
\hline \multirow[t]{2}{*}{$\operatorname{DMT}(\mathrm{n}=17)$} & Pre-treatment & $5.47 \pm 0.94$ & \multirow[t]{2}{*}{$\mathrm{t}=14.20, \quad \mathrm{P}<0.05$} \\
\hline & Post-treatment & $2.00 \pm 0.79$ & \\
\hline \multirow[t]{2}{*}{$\operatorname{PHONO}(\mathrm{n}=14)$} & Pre-treatment & $5.14 \pm 0.77$ & \multirow[t]{2}{*}{$\mathrm{t}=13.47, \quad \mathrm{P}<0.05$} \\
\hline & Post-treatment & $1.93 \pm 0.92$ & \\
\hline \multirow[b]{2}{*}{ CRYO $(n=19)$} & Pre-treatment & $5.21 \pm 1.27$ & \multirow[b]{2}{*}{$\mathrm{t}=7.85, \quad \mathrm{P}<0.05$} \\
\hline & Post-treatment & $2.58 \pm 1.12$ & \\
\hline
\end{tabular}

$\mathrm{P}<0.05=$ Significant difference

Table 4. Cross-comparison of pain perception scores in all groups

\begin{tabular}{|c|c|c|}
\hline \multirow[t]{2}{*}{ Pain Assessment } & \multicolumn{2}{|c|}{ Pain Perception Scores(PPS) } \\
\hline & Mean \pm SD & $\mathrm{P}$ - value \\
\hline Pre-treatment $(\mathrm{DMT}: \mathrm{n}=17)$ & $5.47 \pm 0.94$ & \\
\hline Pre-treatment $($ PHONO $: \mathrm{n}=14)$ & $5.14 \pm 0.77$ & $\begin{array}{l}F=0.44 \\
P>0.05\end{array}$ \\
\hline Pre-treatment $(\mathrm{CRYO}: \mathrm{n}=19)$ & $5.21 \pm 1.27$ & \\
\hline Post-treatment ( DMT $: \mathrm{n}=17$ ) & $2.00 \pm 0.79$ & \\
\hline Post-treatment ( PHONO :n = 14$)$ & $1.93 \pm 0.92$ & $\begin{array}{l}F=2.39 \\
P>0.05\end{array}$ \\
\hline Post-treatment $($ CRYO : $\mathrm{n}=19)$ & $2.58 \pm 1.12$ & \\
\hline
\end{tabular}

$\mathrm{P}>0.05=$ No significant difference

\section{DISCUSSION}

A comparison of the results from the three groups has shown that the difference in the mean treatment sessions was statistically not significant. This result apparently indicates that it might take less treatment sessions to treat subjects with the type of musculoskeletal injuries (MSIs) included in this study if double-modality therapy (DMT) is administered rather than a single treatment protocol of either phonophoresis or cryotherapy. The fewer treatment sessions may be an advantage for the patients with respect to patient waiting time (PWT) and treatment costeffectiveness. Also, considering the pressure to treat athletes who sustain MSIs safely and efficiently in order to get them back to effective performance as quickly as possible, the DMT protocol may have an advantage over other modalities.

The pre-treatment pain perception scores (PPS) compared across the three groups did not show any significant difference, which suggests that all the participants in the three groups had almost the same level of pain perception before the commencement of treatment. A comparison of pre- and post-treatment pain perception scores was made in each group to determine the level of pain relief and the relative efficacy of each modality. The difference in the severity of pain before and after treatment was statistically significant. This shows that each modality (DMT, PHONO, CRYO) was significantly effective in relieving pain and improving function.

There is a dearth in the literature (Balogun, 1990; Ball, 2002) to suggest or indicate the efficacy of combined treatment protocol of phonophoresis and cryotherapy (DMT). Hence, the findings of the current study have provided evidence-based data or guidelines to demonstrate the therapeutic efficacy of DMT protocol in managing musculoskeletal pain. However, to determine whether the DMT protocol would significantly produce pain relief and improve function among the subjects more than the one treatment protocol of either phonophoresis or cryotherapy as suggested in the literature (Santiesteban, 1983; Balogun, 1990), the post-treatment pain perception scores (PPS) across the three groups were compared using ANOVA. There was no statistically significant difference in pain relief. This result implies that the three modalities (DMT, PHONO, CRYO) are equally and significantly effective; the DMT protocol did not produce more pain relief.

\section{CONCLUSION}

The current study has indicated significant therapeutic efficacy of DMT, but it was not superior to the single treatment protocol of either phonophoresis or cryotherapy. The DMT protocol may, however, require less treatment sessions than the single treatment protocols to reduce pain among the type of subjects included in this study.

\section{References}

Allen, L.V. 2005. Compounding for phonophoresis. Secundum Artem 11(2).

Ball, A.M. 2002. Phonophoresis and Ice (PTupdate) [URL=http://www.PTupdate.com]. 
Relative Therapeutic Efficacy of Phonophoresis as Combined Therapy for Musculoskeletal Injuries

Balogun, J.A. 1990. Recent advances in electrotherapy. Journal of Nigeria Association of Sports Science and Medicine 1: 1 - 12.

Beebe, F.A., Barkin, R.L. and Barkin, S. 2005. A clinical and pharmacological review of skeletal muscle relaxants for musculoskeletal conditions. American Journal of Therapeutics 12(2): $151-171$.

Bleakley, C.M., McDonough, S.M. and MacAuley, D.C. 2004. The use of ice in the treatment of acute soft tissue injuries: A systemic review of randomized controlled trials. American Journal of Sports Medicine 32: 251 - 261.

Bleakley, C.M., McDonough, S.M., MacAuley, D.C. and Bjordal, J. 2006. Cryotherapy for acute ankle sprains - a randomised controlled study of two different icing protocols. British Journal of Sports Medicine 40(8): 700 - 705.

Bolin, D.J. 2003. Transdermal approaches to pain in soft tissue injury. Curr Sports Med Rep 2(6): 303 - 309.

Brolinson, P.G. and Sampson, M. 2003. Pathophysiology of pain in sports. Curr Sports Med Rep 2(6): 310 - 314 (Abstract).

Bumin, G. and Can, F. 2004. Effects of iontophoresis and phonophoresis methods on pain cases with shoulder periarthritis. Pain Clinic 13(2): 159 - 162 (Abstract online).

Byl, N.N. 1995. The use of ultrasound as an enhancer for transcutaneous drug delivery: Phonophoresis. Physical Therapy 75(6): 539 - 553.

Cagnie, B.,Vinck, E., Rimbaut, S. and Vanderstraeten, G. 2003. Phonophoresis versustopical application of ketoprofen: comparison between tissue and plasma levels. Physical Therapy 83(8): 707 - 712 .

Childs, J.D., Whitman, J.M., Sizer, P.S., Pugia, M.L., Flynn, T.W. and Delitto,A. 2005. A description of physical therapists' knowledge in managing musculoskeletal conditions. BMC Musculoskeletal Disorders 6: 32 doi: 10.1186/14712474-6-32.

Ciccone, C.D., Leggin, B.G. and Callamaro, J.J. 1991. Effects of ultrasound and trolamine salicylate phonophoresis on delayed -onset muscle soreness. Physical Therapy 71(9): 666 - 675.

Cote, D.J., Prentice, W.E., Hooker, D.N. and Shields, E.W. 1988. Comparison of three treatment procedures for minimizing ankle sprain swelling. Physical Therapy 68(7): 1072 - 1076.

Davis, R.V. 1991. Management of rotator cuff syndrome. Dynamic Chiropractic 9(9): 26-28.

DeRosa, C.P. and Porterfield, J.A. 1992. A physical therapy model for the treatment of low back pain. Physical Therapy 72(4): $261-268$.

Hootman, J.M., Macera, C.A., Ainsworth, B.E., Anddy, C.L, Martin, M. and Blair, S.N. 2002. Epidemiology of musculoskeletal injuries among sedentary and physically active adults. Medicine and Science in Sports and Exercise 34(5): 838 - 844.
Hoppenrath, T. and Ciccone, C.D. 2006. Is there evidence that phonophoresis is more effective than ultrasound in treating pain associated with lateral epicondylitis? Physical Therapy 86(1): 136 - 140 .

Hubbard, T.J., Aronson, S.L. and Denegar, C.R.. 2004. Does cryotherapy hasten return to participation? A systematic review. Journal of Athletic Training 39(1): 88 - 94.

Hubbard, T.J. and Denegar, C.R. 2004. Does cryotherapy improve outcomes with soft tissue injury? Journal of Athletic Training 39(3): 278 - 279.

Idowu, P.A., Adedoyin, R.A. and Adagunodo, R.E. 2005. Computer-related repetitive strain injuries. Journal of Nigeria Society of Physiotherapy 15(1): 13 - 18.

Kellett, J. 1986. Acute soft tissue injuries - a review of the literature. Medicine and Science in Sports and Exercise 18 (5): 489 - 499.

Klaiman, M.D., Shrader, J.A., Danoff, J.V., Hicks, J.E., Pesce, W.J. and Ferland, J. 1998. Phonophoresis versus ultrasound in the treatment of common musculoskeletal conditions. Medicine and Science in Sports and Exercise 30 (9): 1349 1355 (Abstract).

Kozanoglu, E., Basaran, S., Guzel, R. and Guler-Uysal, F. 2003. Short-term efficacy of ibuprofen phonophoresis versus continuous ultrasound therapy in knee osteoarthritis. Swiss Medical Weekly 133: 333 - 338.

Kuntz, A.R., Griffiths, C.M., Rankin, J.M., Amstrong, C.W. and McLaughlin, T.J. 2006. Cortisol concentrations in human skeletal muscle tissue after phonophoresis with $10 \%$ hydrocortisone gel. Journal of Athletic Training 41(3): 321 324.

MacAuley, D.C. 2001. Ice therapy - how good is the evidence? International Journal of Sports Medicine 22(5): 379 - 384.

Machet, L. and Boucaud, A. 2002. Phonophoresis, mechanisms and skin tolerance. International Journal of Pharmaceuticals 243(1-2): 1-15.

Man, I.O., Morrisey, M.C. and Cywinske, J.K. 2007. Effect of nueromuscular electrical stimulation on ankle swelling in the early period after ankle sprain. Physical Therapy 87(1): 401 406.

McElnay, J.C., Benson, H.A.E., Harland, R. and Hadgraft, J. 2004. Phonophoresis of methyl nicotinate: A preliminary study to elucidate the machanism of action. Pharmaceutical Research 10(12): 1726 - 1731 (Abstract online).

Oakley, E.M. 1978. Application of continuous beam ultrasound at therapeutic levels. Physiotherapy 64(6): 169 - 172.

Palmer, T. and Toombs, J.D. 2004. Managing joint pain in primary care. Journal of American Family Practice 17: 532 - 542.

Reilly, T. and Hardiker, R. 1983. Somatotype and injuries in adult student rugby football. Journal of Sports Medicine 12: 186 191. 
Saidu, I.A., Utti, V.A., Jaiyesimi, A.O., Rufa'i, A.A., Maduagwu, S.M., Onuwe, H.A., Jajere, A.M. 2011. Prevalence of musculoskeletal injuries among factory workers in Kano metropolis, Nigeria. International Journal of Occupational Safety and Ergonomics 17(1): 99-102.

Santiesteban, A.J. 1983. The role of physical agents in the treatment of spine pain. Clinical Orthopaedic and Related Research 179: 24 - 29.

Sanya, A.O. and Ogwumike, O.O. 2005. Low back pain prevalence amongst industrial workers in the private sector in Oyo State,
Nigeria. African Journal of Medical Sciences 34: 245 - 249.

Swenson, C., Sward, L. and Karisson, J. 1996. Cryotherapy in sports medicine. Scandinavian Journal of Medicine and Science in Sports 6(4): 193 - 200.

Tyle, P. and Agrawala, P. 1989. Drug delivery by phonophoresis. Pharmaceutical Research 6: 355 - 361.

Wilson, J.J. and Best, T.M. 2005. Common overuse tendon problem: A review and recommendation for treatment. American Family Physician 72(5): 811 - 818. 